\title{
Nonsurgical therapy of chronic periodontitis with adjunctive systemic azithromycin or amoxicillin/metronidazole
}

\author{
Holger F. R. Jentsch ${ }^{1}$ • Andreas Buchmann ${ }^{2} \cdot$ Abel Friedrich $^{2} \cdot$ Sigrun Eick $^{3}$
}

Received: 26 May 2015 / Accepted: 30 November 2015 / Published online: 19 December 2015

(C) Springer-Verlag Berlin Heidelberg 2015

\begin{abstract}
Objectives The objective of the present study is to compare the effect of systemic adjunctive use of azithromycin with amoxicillin/metronidazole to scaling and root planing (SRP) in a clinical study.

Materials and methods Data from 60 individuals with chronic periodontitis were evaluated after full-mouth SRP. Antibiotics were given from the first day of SRP, in the test group $(n=29)$, azithromycin for 3 days and, in the control group $(n=31)$, amoxicillin/metronidazole for7 days. Probing depth (PD), attachment level (AL), and bleeding on probing (BOP) were recorded at baseline and after 3 and 12 months. Gingival crevicular fluid was analyzed for matrix metalloprotease (MMP)- 8 and interleukin (IL)-1beta levels. Subgingival plaque was taken for assessment of the major bacteria associated with periodontitis.

Results In both groups, PD, AL, and BOP were significantly reduced $(p<0.001)$.

A few significant differences between the groups were found; AL and BOP were significantly better in the test
\end{abstract}

Holger F. R. Jentsch and Andreas Buchmann contributed equally to this work.

Holger F. R. Jentsch

jenh@medizin.uni-leipzig.de

1 Centre for Periodontology, Department of Cariology, Endodontology and Periodontology, University Hospital of Leipzig, Liebigstr. 12, Haus 1, 04103 Leipzig, Germany

2 Private dental practice, Weiskirchen, Germany

3 Department of Periodontology, Laboratory of Oral Microbiology, School of Dental Medicine, University of Bern, Bern, Switzerland than in the control group at the end of the study $(p=0.020$ and 0.009$)$. Periodontopathogens were reduced most in the test group.

Conclusions A noninferiority of the treatment with azithromycin in comparison with amoxicillin/metronidazole can be stated. The administration of azithromycin could be an alternative to the use of amoxicillin/metronidazole adjunctive to SRP in patients with moderate or severe chronic periodontitis; however, a randomized placebo-controlled multicenter study is needed.

Clinical relevance Application of azithromycin as a single antibiotic for 3 days might be considered as an additional adjunctive antibiotic to SRP in selected patients.

Keywords Antibiotics - Chronic periodontitis - Cytokines · Periodontopathogens $\cdot$ Scaling and root planing

Scaling and root planing (SRP) is an effective causative method for infection control in the treatment of periodontal disease [1]. Subgingival mechanical debridement results in a mean attachment gain of $2.16 \mathrm{~mm}$ in pockets with an initial depth $\geq 7 \mathrm{~mm}$ [2]. Different systemic and local antimicrobial adjuncts have been shown to improve the outcome of SRP [3-5]. Metronidazole in combination with SRP is more effective than SRP alone in terms of probing depth reduction and clinical attachment level gain [6]. Amoxicillin/metronidazole is an effective adjunct to SRP of chronic periodontitis and an often studied treatment procedure [3]. The clinical and microbiological results of SRP are better with the combination of amoxicillin/metronidazole also in type 2 diabetes patients than without adjunctive antibiotics [7]. At the moment, the use of amoxicillin/metronidazole is largely debated considering multiple aspects of the 
advantages and disadvantages of antibiotics in general and in periodontology [8].

More and more studies evaluated azithromycin as an adjunctive antibiotic in periodontal treatment. Azithromycin is effective against Gram-negative aerobic and anaerobic bacteria, is slowly released to the tissue, and has a long half-life in periodontal tissues [9]. It is a macrolide which inhibits bacterial protein synthesis, quorum sensing, reduces biofilm formation, and modulates the host response [10]. Azithromycin suppresses in vitro the formation and activity of osteoclasts [11], and it diminishes the production of inflammatory mediators in gingival fibroblasts stimulated by Porphyromonas gingivalis LPS [12].

By using a daily single dose of $500 \mathrm{mg}$ for 3 days, higher reduction of probing depth and more attachment level gain than by SRP with placebo have been reported [13-15]. However, there are also studies that did not reveal any additional effect of azithromycin regarding the outcome of SRP [16-19]. Long-lasting beneficial effects of repeated adjunctive azithromycin on gingival inflammation, bleeding on probing (BOP), and tooth mobility were observed up to 192 weeks in an observational clinical study [20].

To our best knowledge, up to now, no study has compared the adjunctive effect of azithromycin to that of amoxicillin and metronidazole in conjunction with nonsurgical periodontal therapy. Therefore, the aim of the present study was to evaluate in a prospective randomized clinical trial over 12 months the two antibiotic regimens by analyzing clinical, biochemical, and microbiological data. The hypothesis to test of this study was that there is inferiority of adjunctive treatment with azithromycin in relation to the treatment with amoxicillin/metronidazole regarding changes of the clinical parameters probing depth (PD), attachment level (AL), and BOP.

\section{Material and methods}

\section{Patients}

Following approval of the study by the Ethics Commission (\#240-2011) of the University of Leipzig Medical Faculty, 96 randomly selected patients were screened and asked to participate in the study. Sixty-two volunteers (29 female and 33 male) gave written and informed consent to their participation in the randomized clinical study in a private dental practice (A.B.). In obtaining the informed consents and in conducting the study, the principles outlined in the Declaration of Helsinki, as revised in 2000, were followed. Only individuals with moderate or severe chronic periodontitis [21] were included in the study. In addition, two other inclusion criteria were at least five sites with PDs $\geq 5 \mathrm{~mm}$ and a minimum of 14 teeth. The interproximal plaque index (API [22]) was required to be $\leq 35 \%$ after two initial prophylaxis and instruction sessions. Results of the API $\leq 35 \%$ reflect a sufficient hygiene to start with periodontal therapy [22]. Individuals were excluded if they had taken antibiotics in the 3 months prior to the study or if they had received periodontal treatment during the previous year. Pregnancy, nursing, smoking, chronic diseases such as diabetes mellitus or rheumatoid arthritis, and allergy to ingredients in the drug were also criteria for exclusion.

All treatment was performed by the same dentist (A.B.). To avoid bias, another investigator blinded to the treatment (A.F.) collected plaque and gingival crevicular fluid (GCF) and assessed the clinical data. A computergenerated randomization table was used to recruit and blindly randomize 62 participants to the either control $(n=31)$ or experimental group $(n=31)$ with a $1: 1$ allocation ratio. Allocation concealment was obtained using a sealed opaque envelope containing the treatment (number of allocation table) to the specific subject. The sealed envelope containing treatment assignment was opened immediately after SRP. All measurements were carried out by a single masked examiner (A.F.). For intraexaminer calibration, the repeated measurements of the PD of one quadrant in ten patients were used for reliability testing resulting in $\kappa$ of 0.91 . He did not perform the SRP and was unaware of the treatment assignment. Documentation was performed by an assistant.

The clinical variables $\mathrm{PD}, \mathrm{AL}$, and $\mathrm{BOP}$ of all teeth were determined in a six-point measurement per tooth (mesiobuccal, buccal, distobuccal, mesiooral, oral, and distooral) with a manual periodontal probe (PCP-UNC 15, Hu-Friedy Manufacturing Co., Chicago, IL, USA) at three appointments: before SRP (baseline, t1), after 3 months (t3), and 12 months (t4). At the same time, samples of the subgingival biofilm and GCF were taken from the deepest site per quadrant. To analyze a shorttime effect on host response, GCF was sampled in addition 14 days after SRP (t2).

For sampling GCF, sterile paper strips (Periopaper; Oraflow Inc., Smithtown, New York, USA) were placed at the entrance of the periodontal pocket for $30 \mathrm{~s}$. This intracrevicular superficial method [23] ensures that the subgingival biofilm in the pocket is not destroyed. Following this, endodontic paper points (ISO 60, Roeko $\mathrm{GmbH}$, Langenau, Germany) were inserted into the pocket until resistance was felt and were left in place for $30 \mathrm{~s}$. The strips were pooled and placed into a tube containing proteinase inhibitors (Sigma-Aldrich, St. Louis, MO, USA). All samples were stored at $-20{ }^{\circ} \mathrm{C}$ immediately after collection and transferred to the laboratory within 1 month where the plaque samples were again stored at $-20^{\circ} \mathrm{C}$ and the GCF samples at $-80{ }^{\circ} \mathrm{C}$ until analysis. 
Fig. 1 Flowchart (adapted to Moher et al. [26]) of the study analyzing the effect of azithromycin in comparison to amoxicillin/metronidazole as an adjunct after scaling and root planing

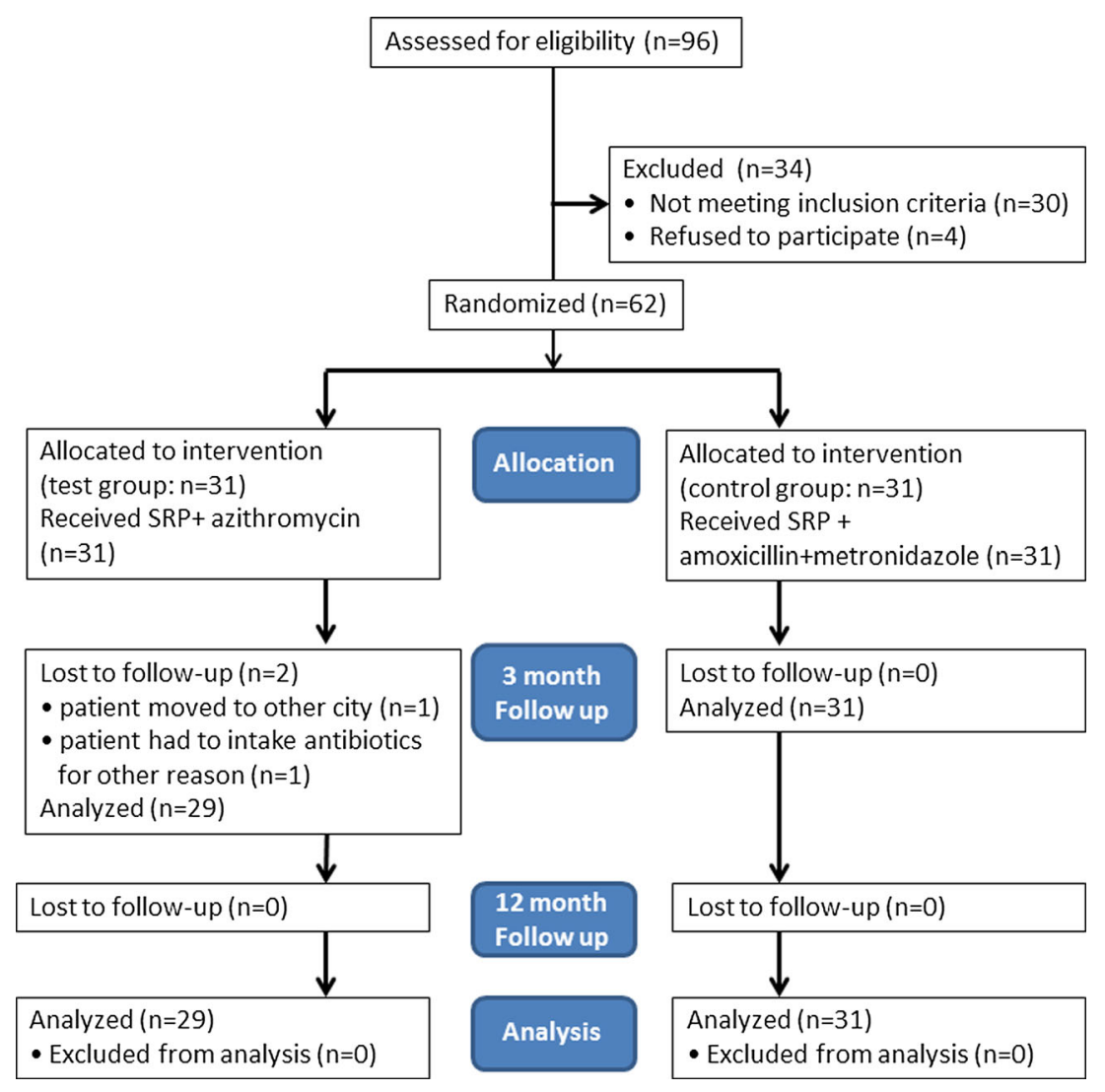

\section{Therapy and follow-up treatment}

Under local anesthesia with articaine hydrochloride/ epinephrine hydrochloride (Ultracain D-S, SanofiAventis, Frankfurt/Main, Germany), the participants received full-mouth SRP in two sessions carried out during two consecutive days using hand and ultrasonic instruments. All of the patients used a chlorhexidine digluconate mouth rinse (Chlorhexamed forte $0.2 \%$, GlaxoSmithKline Healthcare, Bühl, Germany) for $1 \mathrm{~min}$ twice daily during the first 7 days after SRP. Careful normal oral hygiene with toothbrush and interdental brushes was performed.

Antibiotics were given from the first day of SRP. The patients of the test group $(n=31)$ received $500 \mathrm{mg} /$ day azithromycin (azithromycin $500 \mathrm{mg}$, Hexal AG, Holzkirchen, Germany) for 3 days.

Amoxicillin $(3 \times 500 \mathrm{mg} /$ day, Amoxihexal $500 \mathrm{mg}$, Hexal AG, Holzkirchen, Germany) and metronidazole $(3 \times 400 \mathrm{mg} /$ day, metronidazole $400 \mathrm{mg}$, Hexal AG, Holzkirchen, Germany) were administered to the patients of the control group for 7 days. No blinding of the packing and pharmaceutical form has been performed; the drugs were provided by a public pharmacy. During the following 3 or 7 days, a dental assistant of the private practice reminded the patient to take the next or remaining doses. After intake of the tablets, patients were asked to return the tablet packaging to the dental practice.

Every 3 months, careful supportive periodontal therapy was performed by an experienced clinician (A.B.). During the appointment of supportive periodontal care, remotivation and reinstruction have been performed. The patients were controlled for exact drug use. The participants were asked to address the private practice in case of adverse events due to the antibiotic therapy.

\section{Biochemical and microbiological analysis}

Before analyzing, GCF samples were eluted at $4{ }^{\circ} \mathrm{C}$ overnight into $750 \mu \mathrm{l}$ phosphate-buffered saline containing proteinase inhibitors. From the eluates, the levels of interleukin (IL)-1 $\beta$ and matrix metalloprotease (MMP)- 8 were determined by using commercially available enzyme-linked immunosorbent assay (ELISA) kits (R\&D Systems Europe Ltd., Abingdon, UK) according to the manufacturer's instructions. The detection levels of the kits were $2 \mathrm{pg} /$ site for IL- $1 \beta$ and $100 \mathrm{pg} / \mathrm{site}$ for MMP-8.

Microbiological analysis was made as described recently [24]. In short, DNA was extracted by using the Chelex method [25]. Following, the microIDent ${ }^{\circledR}$ plus 11 test (Hain Lifescience, Nehren, Germany) was used according to the manufacturer's description. The test is able to identify 11 
Table 1 Demographic data in the test (azithromycin) and control (amoxicillin/metronidazole) groups at baseline

\begin{tabular}{lll}
\hline Variable & Test group $(n=29)$ & Control group $(n=31)$ \\
\hline Age (years; mean \pm SD) & $54.66 \pm 8.89$ & $53.48 \pm 8.07$ \\
Range (years) & $31-68$ & $32-64$ \\
Male $(n)$ & 13 & 18 \\
Female $(n)$ & 16 & 13 \\
\hline
\end{tabular}

$S D$ standard deviation, $n$ number

periodontopathogenic bacterial species after two PCR runs and a subsequent reverse hybridization. Intensity of staining of the strips was used for semiquantification. Two qualified investigators determined independently the score $(0$ - no band (negative), 1-weak band (low load), 2 - clear band (moderate load), 3-strong band (high load), 4-very strong band (very high load)). Both the reference bands and the hybridization controls were used for adjustment. The qualitative results were used for an additional analysis: the positive results were counted for the major periodontopathogens (Aggregatibacter actinomycetemcomitans, $P$. gingivalis, Tannerella forsythia, and Treponema denticola) and for all analyzed bacterial species (in addition, Parvimonas micra, Fusobacterium nucleatum/necrophorum, Campylobacter rectus, Eubacterium nodatum, Eikenella corrodens, Capnocytophaga sp.).

\section{Data analysis}

The null hypothesis of our study was that there are statistically significant differences in the clinical parameters $\mathrm{PD}, \mathrm{AL}$, and BOP between the test and control groups with inferiority of the test group. Change of PD at the 12-month appointment was set as the primary outcome and used to determine sample size. A mean difference of $1 \mathrm{~mm}$ in observed PD with a standard deviation of $1 \mathrm{~mm}$ between two groups, or two examination dates would require $\geq 16$ patients per group in order to

Table 2 Clinical variables (median, 5 and 95 percentile) at baseline, after 3 months, and 12 months incl. differences (diff.) and statistical analysis in the test (azithromycin) and control (amoxicillin/metronidazole) groups

\begin{tabular}{|c|c|c|c|c|c|}
\hline $\begin{array}{l}N_{\text {test group }}=29 \\
N_{\text {control group }}=31\end{array}$ & Baseline & 3 months & 12 months & $\begin{array}{l}\text { Difference } 3 \\
\text { months-baseline }\end{array}$ & $\begin{array}{l}\text { Difference } 12 \\
\text { months-baseline }\end{array}$ \\
\hline \multicolumn{6}{|l|}{ BOP (\%) } \\
\hline Test group & $45.0(24.0,63.5)$ & $15.0(11.0,25.0)^{* * *}$ & $13.0(10.0,19.0)^{* * *}, \mathbf{q \rrbracket}$ & $29.0(8.5,49.0)$ & $30.0(10.5,50.5) \rrbracket$ \\
\hline Control group & $37.0(30.6,65.0)$ & $15.0(10.2,21.0)^{* * *}$ & $15.0(10.6,21.2)^{* * *}$ & $23.0(14.2,48.0)$ & $22.0(16.0,44.8)$ \\
\hline \multicolumn{6}{|l|}{ GI $(\%)$} \\
\hline Test group & $44.0(26.5,65.0)$ & $21.0(18.0,25.5)^{* * *}$ & $20.0(16.0,22.5)^{* * *}$ & $24.0(5.0,45.0)$ & $26.0(6.0,44.5)$ \\
\hline Control group & $41.0(33.8,64.4)$ & $23.0(17.6,27.8)^{* * *}$ & $20.0(16.6,25.2)^{* * *}$ & $18.0(10.0,43.8)$ & $21.0(13.8,42.2)$ \\
\hline \multicolumn{6}{|l|}{ Median PD (mm) } \\
\hline Test group & $4.07(3.72,4.40)$ & $2.88(2.43,3.32)^{* * *}$ & $2.70(2.24,3.24)^{* * *}$ & $1.20(0.85,1.58)$ & $1.50(1.08,2.67)$ \\
\hline Control group & $4.05(3.43,4.69)$ & $2.90(2.57,3.45)^{* * *}$ & $2.75(2.39,3.33)^{* * *}$ & $1.13(0.82,1.39)$ & $1.28(0.91,1.62)$ \\
\hline \multicolumn{6}{|l|}{ Median AL (mm) } \\
\hline Test group & $4.34(3.86,5.56)$ & $3.20(2.71,4.31)^{* * *}, \boldsymbol{\Phi}$ & $3.04(2.55,4.31)^{* * *}, \mathbb{q}$ & $1.11(0.72,1.49) \uparrow ף$ & $1.18(0.87,1.68) \rrbracket$ \\
\hline Control group & $4.33(3.82,6.41)$ & $3.41(2.98,5.28)^{* * *}$ & $3.30(2.78,5.04)^{* * *}$ & $0.89(0.62,1.28)$ & $1.05(0.72,2.33)$ \\
\hline \multicolumn{6}{|c|}{ Sites PD $\geq 5$ mm $(n)$} \\
\hline Test group & $19.0(5.0,51.0)$ & $0(0,16.0)^{* * *}$ & $0(0,15.5)^{* * *}$ & $18.0(3.5,45.0)$ & $18.0(3.5,45.5)$ \\
\hline Control group & $21.0(5.2,76.8)$ & $0(0,13.2)^{* * *}$ & $0(0,9.6)^{* * *}$ & $21.0(3.2,63.6)$ & $21.0(3.2,67.8)$ \\
\hline \multicolumn{6}{|c|}{ Median PD in sites PD $\geq 5 \mathrm{~mm}$ at baseline (mm) } \\
\hline Test group & $5.14(5.00,5.97)$ & $3.56(3.02,4.48)^{* * *}$ & $3.50(2.75,4.48)^{* * *}$ & $1.62(1.12,2.08)$ & $1.81(1.31,2.71)$ \\
\hline Control group & $5.21(5.00,6.23)$ & $3.67(3.34,4.46)^{* * *}$ & $3.44(3.05,4.35)^{* * *}$ & $1.51(1.08,2.30)$ & $1.76(1.19,2.56)$ \\
\hline \multicolumn{6}{|c|}{ Sites $\mathrm{AL} \geq 5 \mathrm{~mm}(n)$} \\
\hline Test group & $45.0(16.5,108.5)$ & $6.0(0,57.0)^{* * *}, \boldsymbol{\Phi}$ & 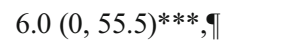 & $33.0(16.5,63.0)$ & $34.0(16.5,64.5)$ \\
\hline Control group & $50.0(21.4,149.0)$ & $9.0(3.0,106.0)^{* * *}$ & $9.0(2.0,97.6)^{* * *}$ & $29.0(17.8,64.2)$ & $34.0(17.8,76.2)$ \\
\hline \multicolumn{6}{|c|}{ Median AL in sites $A L \geq 5 \mathrm{~mm}$ at baseline ( $\mathrm{mm})$} \\
\hline Test group & $5.29(4.63,6.21) \Phi$ & $3.90(3.36,5.05)^{* * *, 9 ף}$ & $3.78(3.24,4.95)^{* * *}$, & $1.34(0.92,1.71) \uparrow ף$ & $1.45(0.98,1.89)$ \\
\hline Control group & $5.48(5.07,6.68)$ & $4.21(3.74,5.53)$ & $4.02(3.46,5.52)^{* * *}$ & $1.150 .76,1.56)$ & $1.36(0.74,2.86)$ \\
\hline
\end{tabular}

$n$ number, $B O P$ bleeding on probing, $G I$ gingival index, $P D$ probing depth, $A L$ attachment level

Wilcoxon' signed rank test for paired samples: ${ }^{*} p<0.05,{ }^{*} p<0.01,{ }^{* *} p<0.001$, each compared with baseline. Mann-Whitney test between the groups: $\boldsymbol{\Phi} p<0.05, \boldsymbol{q} \uparrow<0.01, \boldsymbol{q} \uparrow \uparrow \mid<0.001$ 
Table 3 Biomarkers (median, 5 and 95 percentile) in the test (azithromycin) and control (amoxicillin/metronidazole) groups at baseline, after 14 days as well as 3 and 12 months

\begin{tabular}{llllr}
\hline $\begin{array}{l}N_{\text {test group }}=29 \\
N_{\text {control group }}=31\end{array}$ & Baseline & 14 days & 3 months & 12 months \\
\hline $\begin{array}{l}\text { MMP-8 (ng/site) } \\
\text { Test group }\end{array}$ & $12.33(2.41,38.93)$ & $9.35(0.97,22.65)$ & $11.72(2.22,31.82)$ & $6.97(0.52,32.40)$ \\
$\quad$ Control group & $10.40(1.41,33.85)$ & $7.45(3.43,31.83)$ & $11.85(2.52,33.72)$ & $8.46(3.30,25.39)$ \\
IL-1 $\beta$ (pg/site) & & & & \\
Test group & $31.70(3.06,287.86)$ & $17.18(2.18,155.07)^{*}$ & $48.48(2.50,279.80)$ & $18.52(1.62,496.36)$ \\
Control group & $23.67(4.65,152.25)$ & $23.23(1.69,568.60)$ & $33.62(2.63,257.46)$ & $31.04(2.51,248.95)$ \\
\hline
\end{tabular}

Wilcoxon' signed rank test for paired samples

${ }^{*} p<0.05$ compared with baseline

detect a significant difference $(p \leq 0.05)$ with a test power of $80 \%$ (GraphPad StatMate v.2.0 for Mac, GraphPad Software, San Diego, CA, USA). Secondary outcome variables were changes of PD after 3 months, in the number of sites with $\mathrm{PD} \geq 5 \mathrm{~mm}$, occurrence of BOP, mean AL, levels of MMP- 8 and IL- $1 \beta$, and the counts of selected pathogenic bacteria associated with periodontitis after 3 and 12 months.

Statistical analysis of the clinical and laboratory data was performed using the software SPSS ${ }^{\circledR}$ Statistics 21.0 (IBM Corporation, New York, NY, USA). Unit of analysis in all statistical tests was the individual. For both intragroup and intergroup comparisons, nonparametric tests (Wilcoxon test for paired samples and Mann-Whitney $U$ test, respectively) were used. A level of $\alpha \leq 0.05$ was considered as being significant.

\section{Results}

The study was performed in 2012 and 2013. Figure 1 presents the study flow adapted to Moher et al. [26]. The demographic data of these 60 patients are summarized in Table 1.

No severe adverse effects of the different antibiotics were observed during the study. No significant differences were seen at API. Changes of the clinical data during the study are presented in Table 2. BOP was not significantly different between the groups after 3 months but, after 12 months, lower in the test group than in the controls $(p=0.009)$. The changes of BOP were significantly higher in the test group during the whole study period $(p<0.001)$. AL was significantly better in the test group at the end of the study $(p=0.020)$. The mean change of PD after 12 months and the change of AL during the first 3 months and during the whole study period were significantly higher in the test group than in the controls $(p=0.001, p=0.001$, $p=0.021)$. There were significantly fewer sites with $\mathrm{AL}$ $\geq 5 \mathrm{~mm}$ after 3 and 12 months in the test group in comparison with the control group ( $p=0.019, p=0.037$ ).
The levels of MMP-8 and IL-1 $\beta$ are given in Table 3. No significant differences occurred at MMP-8.

The analysis of sums of positive results for analyzed bacteria revealed no significant difference between the test and control groups at baseline. After 3 and 12 months, lower numbers of positive results were detected in the test group than in the control group (Table 4). There were also no significant differences between the test and control groups at the semiquantitative analysis of 11 tested bacterial species at baseline. Of the major periodontopathogens, lower counts of T. denticola and Prevotella intermedia were detected in the test group than in the control group ( $p=0.031,0.016)$ after 3 months. After 12 months, still, the counts of $T$. denticola were significantly lower in the test group ( $p=0.002$, Fig. 2).

\section{Discussion}

This study analyzed the effects of two different regimes of adjunctive administration of systemic antibiotics during SRP in cases with moderate to severe chronic periodontitis on clinical, biochemical, and microbiological results. To our best knowledge and literature search, such a study approach has not been published until now. The test hypothesis can be rejected, and inferiority of adjunctive antibiotic treatment with azithromycin was not observed. The change of mean PD after 12 months was set as the primary outcome. The difference between the groups was only $0.32 \mathrm{~mm}$ in median (significance in favor of azithromycin). From the clinical point of view, PD, $\mathrm{AL}$, and BOP were improved in both groups at every followup appointment underlining the efficacy of both treatments. Favorable 12-month results for adjunctive azithromycin in comparison to SRP only were reported for the treatment of aggressive periodontitis; here, the difference of mean PD difference was about $1 \mathrm{~mm}$ [14]. Comparing different adjunctive treatment regimens in chronic periodontitis azithromycin was as effective as metronidazole in reducing mean PD [27] or did 
Table 4 Numbers (\% per group) of the positive results of major periodontopathogenic bacteria (A. actinomycetemcomitans, $P$. gingivalis, T. forsythia, $T$. denticola) and of the 11 analyzed species (in addition, $P$. intermedia, $P$. micra, F. nucleatum/necrophorum, C. rectus, E. nodatum, E. corrodens, Capnocytophaga sp.) in the test (azithromycin) and control (amoxicillin/metronidazole) groups at baseline as well as 3 and 12 months after SRP with adjunctive azithromycin (test) and with amoxicillin/metronidazole (control) incl. statistics ( $p$ values)

\begin{tabular}{|c|c|c|c|c|c|c|}
\hline & Baseline & 3 months & 12 months & $\begin{array}{l}\text { All time- } \\
\text { points }(p)\end{array}$ & $\begin{array}{l}3 \text { months- } \\
\text { baseline }(p)\end{array}$ & $\begin{array}{l}12 \text { months- } \\
\text { baseline }(p)\end{array}$ \\
\hline \multicolumn{7}{|c|}{ Major periodontopathogens } \\
\hline \multicolumn{7}{|l|}{ Test group $(n=29)$} \\
\hline No detection & $4(13.8)$ & $9(31.0)$ & $9(31.0)$ & 0.028 & 0.044 & 0.039 \\
\hline 1 species & $5(17.2)$ & $7(24.1)$ & $7(24.1)$ & & & \\
\hline 2 species & $6(20.7)$ & $6(20.7)$ & $8(27.6)$ & & & \\
\hline 3 species & $13(44.8)$ & $7(24.1)$ & $4(13.8)$ & & & \\
\hline 4 species & $1(3.4)$ & 0 & $1(3.4)$ & & & \\
\hline \multicolumn{7}{|c|}{ Control group $(n=31)$} \\
\hline No detection & $1(3.2)$ & $5(16.1)$ & $6(19.4)$ & 0.412 & 0.528 & 0.220 \\
\hline 1 species & 7 (22.6) & $2(6.5)$ & $4(12.9)$ & & & \\
\hline 2 species & $5(16.1)$ & $10(32.3)$ & $8(25.8)$ & & & \\
\hline 3 species & $18(58.1)$ & $13(41.9)$ & $11(35.5)$ & & & \\
\hline 4 species & 0 & $1(3.2)$ & $2(6.5)$ & & & \\
\hline Test vs. control $(p)$ & 0.470 & 0.023 & 0.047 & & & \\
\hline \multicolumn{7}{|c|}{ Analyzed 11 periodontopathogens } \\
\hline \multicolumn{7}{|l|}{ Test group $(n=29)$} \\
\hline No detection & 0 & $2(6.9)$ & $1(3.4)$ & 0.015 & 0.016 & 0.047 \\
\hline $1-2$ species & $2(6.9)$ & $4(13.8)$ & $4(13.8)$ & & & \\
\hline $3-4$ species & $7(24.1)$ & $10(34.5)$ & $12(41.4)$ & & & \\
\hline $5-6$ species & $11(37.9)$ & $10(34.5)$ & $8(27.6)$ & & & \\
\hline$\geq 7$ species & $9(31.0)$ & $3(10.3)$ & $4(13.8)$ & & & \\
\hline \multicolumn{7}{|c|}{ Control group $(n=31)$} \\
\hline No detection & 0 & $1(3.2) 1$ & 0 & 0.364 & 0.183 & 0.229 \\
\hline $1-2$ species & $1(3.2)$ & $1(3.2)$ & $2(6.5)$ & & & \\
\hline 3-4 species & $8(25.8)$ & $9(29.0)$ & $8(25.8)$ & & & \\
\hline $5-6$ species & $8(25.8)$ & $9(29.0)$ & $10(32.3)$ & & & \\
\hline$\geq 7$ species & $14(45.2)$ & $11(35.5)$ & $11(35.5)$ & & & \\
\hline Test vs. control $(p)$ & 0.520 & 0.024 & 0.039 & & & \\
\hline
\end{tabular}

not show significant differences to exclusive SRP only after 1 year [17].

The advantage of the use of azithromycin is the short course of administration of the antibiotic which may contribute to the patients' compliance. We did not see any side effect, and no patient stopped the intake of either azithromycin or the combination of amoxicillin and metronidazole.

A systematic review calculated a mean reduction of $1.41 \mathrm{~mm}$ for PD and of $0.94 \mathrm{~mm}$ for AL after SRP with adjunctive amoxicillin/metronidazole [4]. In comparison to these results, our study revealed $1.3-\mathrm{mm}$ improvement of $\mathrm{PD}$ and 1.1-mm attachment gain for the control group with adjunctive amoxicillin/metronidazole. These results are reasonable for SRP in a private dental practice. Most studies on SRP with and without adjunctive antibiotics have been conducted at universities. The conditions there may differ to a certain extent from those in private dental practices. Further attachment loss is reduced when patients are treated at universities conditions and when regular supportive periodontal care is provided [28]. During the supportive periodontal therapy, a better stability of $\mathrm{AL}$ was found by specialist care as compared to general practice [29]. In our study, the treatment was performed by a dentist with special interest in periodontology in a general dental practice. It should be taken into account that this is the normal clinical situation for the most patients. In general, the analyzed bacteria were more reduced after adjunctive azithromycin than after the combined administration of amoxicillin and metronidazole. After 3 and 12 months, $T$. denticola was found to be significantly lower in the 

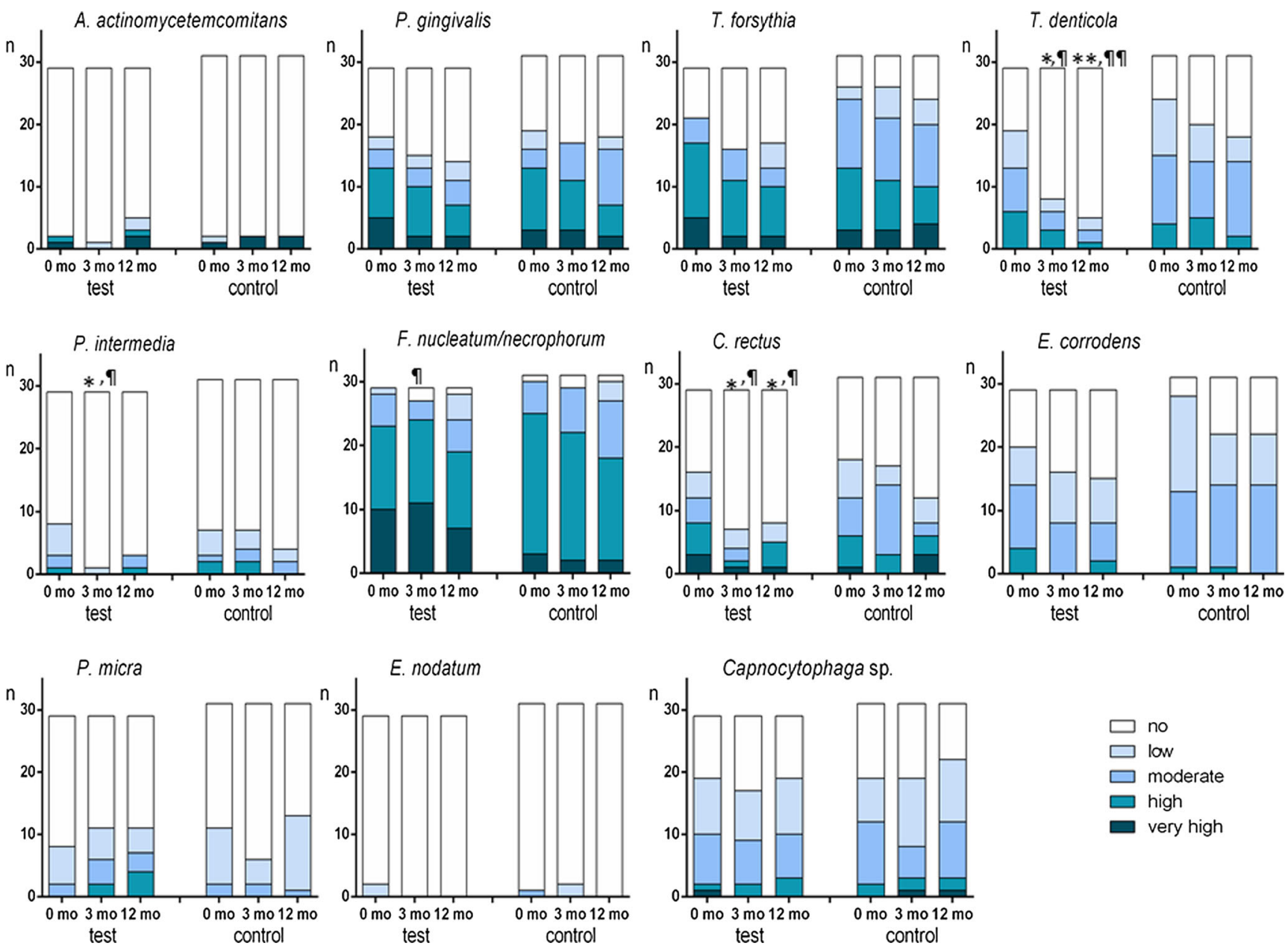

Fig. 2 Semiquantitative loads of periodontopathogenic bacteria ( $A$. actinomycetemcomitans, $P$. gingivalis, T. forsythia, T. denticola, $P$. intermedia, $F$. nucleatum/necrophorum, $C$. rectus, E. corrodens, $P$. micra, E. nodatum, Capnocytophaga sp.) at baseline as well as 3 and

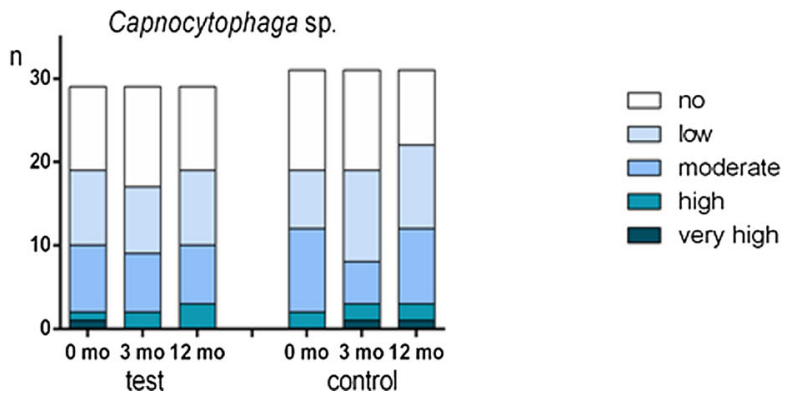

12 months after SRP with adjunctive azithromycin (test) and amoxicillin/metronidazole (control). $\uparrow p<0.05, \uparrow \uparrow p<0.01$ significant intergroup difference, ${ }^{*} p<0.05, * * p<0.01$ significant difference to baseline

subgingival biofilm in the test group. This finding is important because $T$. denticola is strongly related to further periodontal breakdown in periodontitis and to the recurrence of the disease [30]. T. denticola lives in metabolic symbioses with $P$. gingivalis [31] and is difficult to suppress by combined mechanical and antibiotic therapy [32]. Azithromycin activity is not limited on killing bacteria; it decreases also the bacterial virulence. In subinhibitory concentrations, it inhibits the fimbrial production of $P$. gingivalis [33], reduces biofilm formation, and decreases the counts of $P$. gingivalis within the biofilm [34]. Azithromycin is of interest for the treatment of Pseudomonas aeruginosa infections. Although not acting growth inhibitory, it suppresses the expression of virulence factors, biofilm formation, and quorum sensing [35].

On the other hand, azithromycin interferes with immune cells. In our study, the levels of the inflammatory markers MMP- 8 and IL-1 $\beta$ were determined immediately 14 days

up to 1 year after starting the intake of the antibiotics. IL$1 \beta$ belonging to the IL-1 cytokines is induced by proinflammatory agents such as lipopolysaccharide, TNF, and IL-1 $\beta$ itself; it accounts for neutrophil infiltration and tissue turnover via inducing matrix metalloproteinases (MMPs) [36]. MMPs as a family of enzymes which degrade extracellular matrix and basement membrane components are mostly produced in a latent form; activation most involves tissue, plasma, and bacterial proteinases together with oxidative stress [37]; MMP-8 (neutrophil collagenase) targets native collagen [38].

The only striking result was seen after 14 days in the test group; here, the levels of IL- $1 \beta$ decreased significantly $(p=0.030)$ and those of MMP- 8 by trend $(p=0.061)$ suggesting an influence of azithromycin on host cells. In vitro, the uptake of azithromycin by monocytic cells leads to an enhanced release of inflammatory mediators (e.g., IL-1 $\beta$ ) and reduced killing which are reversed after $24 \mathrm{~h}$ [39]. The mRNA 
expression and following the protein levels of IL-10 are increased dose-dependently by azithromycin in activated monocytes [40]. In healthy volunteers, it was shown that IL-8 and IL-1 $\beta$ levels increased up to $24 \mathrm{~h}$ after intake of azithromycin and returned to baseline after 28 days [41]. Azithromycin reduces the amount of GCF as well as several cytokines (IL$1 \beta$, IL- 8, TNF- $\alpha$ ) in the GCF [42]. Our results are comparable to those of another study in chronic periodontitis patients, where MMP-8 levels decreased immediately 14 days after treatment, while no difference to baseline was found 3 months later [17].

Our study has limitations. No negative control, an SRP without adjunctive antibiotic treatment, was included into the study. Although the examiner was not aware of the treatment groups, the drugs were not blinded to the clinician and no placebo was used. Inclusion criteria were at least five sites with $\mathrm{PD} \geq 5 \mathrm{~mm}$. However, it should be mentioned that the majority of the sites with $\geq 5 \mathrm{~mm}$ had a PD of $5 \mathrm{~mm}$. This might influence the favorable results regarding azithromycin. The results of this one-center study need to be confirmed in a randomized placebo-controlled multicenter trial.

Although comparing here two groups with adjunctive antibiotics, the use should be limited to selected cases. An absolute precondition is a well-performed mechanical plaque control since it is well known that antibiotics are less active within biofilms [43, 44]. Irrespective of this, a global problem is the development of resistance which is clearly associated with the consumption of antibiotics [45].

Within in the limits of the present study, it can be summarized that azithromycin might be an alternative when an adjunctive antibiotic treatment of moderate to severe chronic periodontitis during SRP is needed.

Acknowledgments The authors appreciate the laboratory work of Stéphanie Larti, Anna Magdon, and Marianne Weibel. They are grateful to Timothy Jones (Institute of Applied Linguistics and Translatology, University of Leipzig) for proofreading and to Walter B. Bürgin, Dipl. Biomed. Ing. (University of Bern), for statistical support.

\section{Compliance with ethical standards}

Conflict of interest The authors (Holger Jentsch, Andreas Buchmann, Abel Friedrich, and Sigrun Eick) declare that they have no conflict of interest.

Funding The study was funded by the participating institutions and partly supported by Hain Diagnostica, Nehren, Germany, who provided the materials for microbiological laboratory analyses.

Ethical approval All procedures performed in studies involving human participants were in accordance with the ethical standards of the institutional and/or national research committee and with the 1964 Helsinki declaration and its later amendments or comparable ethical standards.

Informed consent Informed consent was obtained from all individual participants included in the study.

\section{References}

1. Apatzidou DA, Kinane DF (2010) Nonsurgical mechanical treatment strategies for periodontal disease. Dent Clin North Am 54:112. doi:10.1016/j.cden.2009.08.006S0011-8532(09)00071-8

2. Cobb CM (2002) Clinical significance of non-surgical periodontal therapy: an evidence-based perspective of scaling and root planing. J Clin Periodontol 29(Suppl 2):6-16

3. Sgolastra F, Gatto R, Petrucci A, Monaco A (2012) Effectiveness of systemic amoxicillin/metronidazole as adjunctive therapy to scaling and root planing in the treatment of chronic periodontitis: a systematic review and meta-analysis. J Periodontol 83:1257-1269. doi:10. 1902/jop.2012.110625

4. Zandbergen D, Slot DE, Cobb CM, Van der Weijden FA (2013) The clinical effect of scaling and root planing and the concomitant administration of systemic amoxicillin and metronidazole: a systematic review. J Periodontol 84:332-351. doi:10.1902/jop.2012. 120040

5. Aljateeli M, Giannobile WV, Wang HL (2013) Locally-delivered antibiotics for management of periodontitis: current understanding. J Mich Dent Assoc 95:42-47

6. Sgolastra F, Severino M, Petrucci A, Gatto R, Monaco A (2014) Effectiveness of metronidazole as an adjunct to scaling and root planing in the treatment of chronic periodontitis: a systematic review and meta-analysis. J Periodontal Res 49:10-19. doi:10.1111/ jre.12089

7. Miranda TS, Feres M, Perez-Chaparro PJ, Faveri M, Figueiredo LC, Tamashiro NS, Bastos MF, Duarte PM (2014) Metronidazole and amoxicillin as adjuncts to scaling and root planing for the treatment of type 2 diabetic subjects with periodontitis: 1-year outcomes of a randomized placebo-controlled clinical trial. J Clin Periodontol 41:890-899. doi:10.1111/jcpe.12282

8. Preus HR, Scheie AA, Baelum V (2014) Letter to the editor: Re: The clinical effect of scaling and root planing and the concomitant administration of systemic amoxicillin and metronidazole: a systematic review; Re: Effectiveness of systemic amoxicillin/ metronidazole as adjunctive therapy to scaling and root planing in the treatment of chronic periodontitis: a systematic review and meta-analysis; Re: Effectiveness of systemic amoxicillin/ metronidazole as an adjunctive therapy to full-mouth scaling and root planing in the treatment of aggressive periodontitis: a systematic review and meta-analysis. J Periodontol 85:374-384. doi:10. 1902/jop.2014.130379

9. Muniz FW, de Oliveira CC, de Sousa CR, Moreira MM, de Moraes ME, Martins RS (2013) Azithromycin: a new concept in adjuvant treatment of periodontitis. Eur J Pharmacol 705:135-139. doi:10. 1016/j.ejphar.2013.02.044S0014-2999(13)00153-2

10. Parnham MJ, Erakovic Haber V, Giamarellos-Bourboulis EJ, Perletti G, Verleden GM, Vos R (2014) Azithromycin: mechanisms of action and their relevance for clinical applications. Pharmacol Ther 143:225-245. doi:10.1016/j.pharmthera.2014.03.003S01637258(14)00055-2

11. Gannon SC, Cantley MD, Haynes DR, Hirsch R, Bartold PM (2013) Azithromycin suppresses human osteoclast formation and activity in vitro. J Cell Physiol 228:1098-1107. doi:10.1002/jcp. 24259

12. Doyle CJ, Fitzsimmons TR, Marchant C, Dharmapatni AA, Hirsch R, Bartold PM (2015) Azithromycin suppresses $P$. gingivalis LPSinduced pro-inflammatory cytokine and chemokine production by human gingival fibroblasts in vitro. Clin Oral Investig 19:221-227. doi:10.1007/s00784-014-1249-7

13. Oteo A, Herrera D, Figuero E, O’Connor A, Gonzalez I, Sanz M (2010) Azithromycin as an adjunct to scaling and root planing in the treatment of Porphyromonas gingivalis-associated periodontitis: a 
pilot study. J Clin Periodontol 37:1005-1015. doi:10.1111/j.1600051X.2010.01607.xCPE1607

14. Haas AN, de Castro GD, Moreno T, Susin C, Albandar JM, Oppermann RV, Rosing CK (2008) Azithromycin as an adjunctive treatment of aggressive periodontitis: 12-months randomized clinical trial. J Clin Periodontol 35:696-704. doi:10.1111/j.1600-051X. 2008.01254.xCPE1254

15. Yashima A, Gomi K, Maeda N, Arai T (2009) One-stage full-mouth versus partial-mouth scaling and root planing during the effective half-life of systemically administered azithromycin. J Periodontol 80:1406-1413. doi:10.1902/jop.2009.090067

16. Haas AN, Seleme F, Segatto P, Susin C, Albandar J, Oppermann RV, Fontanella VR, Rosing CK (2012) Azithromycin as an adjunctive treatment of aggressive periodontitis: radiographic findings of a 12-month randomized clinical trial. Am J Dent 25:215-219

17. Han B, Emingil G, Ozdemir G, Tervahartiala T, Vural C, Atilla G, Baylas H, Sorsa T (2012) Azithromycin as an adjunctive treatment of generalized severe chronic periodontitis: clinical, microbiologic, and biochemical parameters. J Periodontol 83:1480-1491. doi:10. 1902/jop.2012.110519

18. Emingil G, Han B, Ozdemir G, Tervahartiala T, Vural C, Atilla G, Baylas H, Sorsa T (2012) Effect of azithromycin, as an adjunct to nonsurgical periodontal treatment, on microbiological parameters and gingival crevicular fluid biomarkers in generalized aggressive periodontitis. J Periodontal Res 47:729-739. doi:10.1111/j.16000765.2012.01488.x

19. Sampaio E, Rocha M, Figueiredo LC, Faveri M, Duarte PM, Gomes Lira EA, Feres M (2011) Clinical and microbiological effects of azithromycin in the treatment of generalized chronic periodontitis: a randomized placebo-controlled clinical trial. J Clin Periodontol 38:838-846. doi:10.1111/j.1600-051X.2011.01766.x

20. Schmidt E, Kaciroti N, Loesche W (2011) Benefits of additional courses of systemic azithromycin in periodontal therapy. Gen Dent 59:180-187, quiz 188-9

21. Armitage GC (1999) Development of a classification system for periodontal diseases and conditions. Ann Periodontol 4:1-6. doi: 10.1902/annals.1999.4.1.1

22. Lange DE, Plagmann HC, Eenboom A, Promesberger A (1977) Clinical methods for the objective evaluation of oral hygiene. Dtsch Zahnarztl Z 32:44-47

23. Griffiths GS (2003) Formation, collection and significance of gingival crevice fluid. Periodontol 2000(31):32-42

24. Eick S, Straube A, Guentsch A, Pfister W, Jentsch H (2011) Comparison of real-time polymerase chain reaction and DNAstrip technology in microbiological evaluation of periodontitis treatment. Diagn Microbiol Infect Dis 69:12-20. doi:10.1016/j. diagmicrobio.2010.08.017S0732-8893(10)00346-9

25. Yang JL, Wang MS, Cheng AC, Pan KC, Li CF, Deng SX (2008) A simple and rapid method for extracting bacterial DNA from intestinal microflora for ERIC-PCR detection. World J Gastroenterol 14: 2872-2876

26. Moher D, Schulz KF, Altman DG (2001) The CONSORT statement: revised recommendations for improving the quality of reports of parallel-group randomised trials. Lancet 357:1191-1194

27. Haffajee AD, Torresyap G, Socransky SS (2007) Clinical changes following four different periodontal therapies for the treatment of chronic periodontitis: 1-year results. J Clin Periodontol 34:243253. doi:10.1111/j.1600-051X.2006.01040.x

28. Axelsson P, Lindhe J (1981) The significance of maintenance care in the treatment of periodontal disease. J Clin Periodontol $8: 281-294$

29. Gaunt F, Devine M, Pennington M, Vernazza C, Gwynnett E, Steen N, Heasman P (2008) The cost-effectiveness of supportive periodontal care for patients with chronic periodontitis. J Clin Periodontol 35:67-82. doi:10.1111/j.1600-051X.2008.01261. xCPE1261
30. Meyer-Baumer A, Eick S, Mertens C, Uhlmann L, Hagenfeld D, Eickholz P, Kim TS, Cosgarea R (2014) Periodontal pathogens and associated factors in aggressive periodontitis: results 5-17 years after active periodontal therapy. J Clin Periodontol 41:662-672. doi: $10.1111 /$ jcpe. 12255

31. Tan KH, Seers CA, Dashper SG, Mitchell HL, Pyke JS, Meuric V, Slakeski N, Cleal SM, Chambers JL, McConville MJ, Reynolds EC (2014) Porphyromonas gingivalis and Treponema denticola exhibit metabolic symbioses. PLoS Pathog 10:e1003955. doi:10.1371/ journal.ppat.1003955PPATHOGENS-D-13-02117

32. Ramich T, Schacher B, Scharf S, Rollke L, Arndt R, Eickholz P, Nickles K (2015) Subgingival plaque sampling after combined mechanical and antibiotic nonsurgical periodontal therapy. Clin Oral Investig 19:27-34. doi:10.1007/s00784-014-1208-3

33. Lo Bue AM, Rossetti B, Cali G, Nicoletti G, Condorelli F (1997) Antimicrobial interference of a subinhibitory concentration of azithromycin on fimbrial production of Porphyromonas gingivalis. J Antimicrob Chemother 40:653-657

34. Maezono H, Noiri Y, Asahi Y, Yamaguchi M, Yamamoto R, Izutani N, Azakami H, Ebisu S (2011) Antibiofilm effects of azithromycin and erythromycin on Porphyromonas gingivalis. Antimicrob Agents Chemother 55:5887-5892. doi:10.1128/AAC.0516911AAC.05169-11

35. Imperi F, Leoni L, Visca P (2014) Antivirulence activity of azithromycin in Pseudomonas aeruginosa. Front Microbiol 5: 178. doi:10.3389/fmicb.2014.00178

36. Barksby HE, Lea SR, Preshaw PM, Taylor JJ (2007) The expanding family of interleukin-1 cytokines and their role in destructive inflammatory disorders. Clin Exp Immunol 149:217-225. doi:10. 1111/j.1365-2249.2007.03441.x

37. Sorsa T, Tjaderhane L, Salo T (2004) Matrix metalloproteinases (MMPs) in oral diseases. Oral Dis 10:311-318. doi:10.1111/j. 1601-0825.2004.01038.x

38. Uitto VJ (2000) Overall CM and McCulloch C (2003) Proteolytic host cell enzymes in gingival crevice fluid. Periodontol 31:77-104

39. Hall IH, Schwab UE, Ward ES, Butts JD, Wolford ET, Ives TJ (2002) Disposition and intracellular activity of azithromycin in human THP-1 acute monocytes. Int J Antimicrob Agents 20:348-360

40. Polancec DS, Munic Kos V, Banjanac M, Vrancic M, Cuzic S, Belamaric D, Parnham MJ, Polancec D, Erakovic Haber V (2012) Azithromycin drives in vitro GM-CSF/IL-4-induced differentiation of human blood monocytes toward dendritic-like cells with regulatory properties. J Leukoc Biol 91:229-243. doi:10.1189/j1b. 1210655jlb.1210655

41. Culic O, Erakovic V, Cepelak I, Barisic K, Brajsa K, Ferencic Z, Galovic R, Glojnaric I, Manojlovic Z, Munic V, Novak-Mircetic R, Pavicic-Beljak V, Sucic M, Veljaca M, Zanic-Grubisic T, Parnham MJ (2002) Azithromycin modulates neutrophil function and circulating inflammatory mediators in healthy human subjects. Eur J Pharmacol 450:277-289

42. Ho W, Eubank T, Leblebicioglu B, Marsh C, Walters J (2010) Azithromycin decreases crevicular fluid volume and mediator content. J Dent Res 89:831-835. doi:10.1177/ 00220345103686500022034510368650

43. Belibasakis GN, Thurnheer T (2014) Validation of antibiotic efficacy on in vitro subgingival biofilms. J Periodontol 85:343-348. doi:10.1902/jop.2013.130167

44. Tsaousoglou P, Nietzsche S, Cachovan G, Sculean A, Eick S (2014) Antibacterial activity of moxifloxacin on bacteria associated with periodontitis within a biofilm. J Med Microbiol 63:284-292. doi: 10.1099/jmm.0.065441-0jmm.0.065441-0

45. Bell BG, Schellevis F, Stobberingh E, Goossens H, Pringle M (2014) A systematic review and meta-analysis of the effects of antibiotic consumption on antibiotic resistance. BMC Infect Dis 14:13. doi:10.1186/1471-2334-14-131471-2334-14-13 